\title{
Supersymmetric Partners of Oblique Corrections ${ }^{a}$
}

\author{
L. Randall, E. Katz and S. Su \\ Center for Theoretical Physics, \\ Laboratory for Nuclear Science and Department of Physics \\ Massachusetts Institute of Technology \\ Cambridge, MA 02139, USA
}

\begin{abstract}
We discuss a potential new probe of supersymmetric physics. In particular, we discuss the possibility of measuring hard supersymmetry violation which occurs at one loop through "super-oblique" corrections to the gauge and gaugino propagators. In models with heavy scalar partners, or with many gauge-charged particles which participate in supersymmetry breaking, these effects can be substantial due to logarithmic and multiplicity factor enhancements.
\end{abstract}

\section{Introduction}

Supersymmetry has yet to be discovered. If/When it is, the information about the underlying theory will be limited but powerful. For example, the spectrum of superpartners might tell us about the mechanism of communication of supersymmetry breaking or consistency with a specific high-energy model (GUT theory for example). However, these measurements are (probably) a long way off. In the meantime, it is useful to be prepared to interpret what will be measured. This might be through new insights into the fundamental underlying theory, new models of the effective low-energy physics, or by new ideas for interesting quantities to measure, and how to interpret them.

In this talk, I will discuss a new category of precision measurements which one might use to probe supersymmetric theories. As we will discuss, these measurements have much in common with the well-studied "oblique" corrections which characterized corrections to the standard model. In this case, the measurements will be more difficult, but should they prove possible, could potentially yield invaluable insights into the underlying physics. The york we discuss today will be presented in more detail in a future publication 1 .

The key to understanding the quantities we wish to consider is to distinguish hard and soft supersymmetry breaking parameters. The focus of most research on supersymmetric phenomenology focusses on the soft supersymmetry breaking parameters, in particular the superpartner masses. Hard supersymmetry breaking, on the other hand, is "forbidden". However, this is not true, even in the softly broken supersymmetric theory! The only con-

${ }^{a}$ Talk presented by L. Randall at the SUSY97 Conference, May 27-31, Philadelphia PA, USA. 
straint is that the coefficients of hard supersymmetry breaking operators are finite. There are no available counterterms in the supersymmetric Lagrangian. Nonetheless, finite corrections are permissible and present.

Our focus here is on new parameters which reflect hard supersymmetry breaking in the form of oblique corrections in the gauge sector, akin to precision electroweak parameters. Recall the merits of precision electroweak paramters. They are new parameters which reflect the spontaneous breaking of the elcectroweak gauge symmetry. They served as tests of the standard model, and gave insights into non-standard model physics. Similarly, new SUSY-oblique corrections will test the MSSM, and serve as probes of new physics.

\section{Oblique and Super-Oblique}

It is therefore useful to review standard oblique corrections. Precision electroweak corrections were important in that they tested consistency of the standard model and gave insight into the high-energy world. The oblique corrections are the corrections to the gauge boson propagator. The parameters which characterize the most important effects can be obtained by retaining the leading pieces in a derivative expansion, accounting for gauge invariance. The six "parameters" are $\Pi_{\gamma \gamma}^{\prime}, \Pi_{\gamma Z}^{\prime}, \Pi_{W W}, \Pi_{W W}^{\prime}, \Pi_{Z Z}, \Pi_{Z Z}^{\prime}$. We chose to work in the basis of tree-level mass eigenstates and retained only the nondecoupling pieces of the propagator, those of dimension four or less. These are not the six parameters which were actually used when comparing to measurements; it is more useful first to absorb three of the parameters into $g, g^{\prime}, v$. We are left with three new finite parameters which are forbidden by gauge invariance but permitted once it is spontaneously broken. With my collaborator Mitch Golden, we identified these three parameters as $2 \delta Z_{Z Z}, \delta Z_{\gamma Z}, \delta m_{Z Z}$. In the more widely utilized Peskin-Takeuchi naming convention, they were absorbed into parameters $3 . S, T_{H} U$, while a third popular convention from a paper of Altarelli and Barbieri 5 absorbed them in the three parameters $\epsilon_{1}, \epsilon_{2}, \epsilon_{3}$. The importance of these parameters is that even heavy states do not decouple. For example, one can compute the contributions of a heavy doublet to the $S$ parameter, $S=-\left.4 \pi \frac{d}{d q^{2}} \Pi_{3 S}\left(q^{2}\right)\right|_{q^{2}=0}$

$$
\Delta S=\frac{1}{6 \pi}\left[1-Y \log \left(\frac{m_{N}^{2}}{m_{E}^{2}}\right)\right] .
$$

Of course, the many new particles present in a supersymmetric theory can contribute to the standard oblique corrections. In general, for reasonable values of the supersymmetric partner masses, these effects are quite small. However, these are not what we wish to discuss here. We instead focus on 
a new class of oblique corrections which one can define in supersymmetric theories. Supersymmetry guarantees equality of the gauge and gaugino Yukawa couplings. When supersymmetry is broken, these are no longer guaranteed to be equal. It is easy to calculate the one-loop effect of a single heavy doublet superfield to the difference of $\mathrm{SU}(2)$ gauge and $\mathrm{SU}(2)$ gaugino Yukawa coupling by calculating the "super-oblique" correction to the gauge propagator and gaugino propagator. One thereby derives

$$
\frac{g-\tilde{g}}{g}=\frac{\alpha}{24 \pi}\left(\log \left(\frac{m_{\tilde{W}}}{m_{\tilde{q}}}\right)^{2}-\frac{11}{12}\right)
$$

Here we have computed the contribution of a heavy doublet squark to the difference in $\mathrm{SU}(2)$ gauge vs. gaugino coupling. For physical quarks, they are generally lighter than the gaugino, so we have cutoff the logarithm at the gaugino mass. We have also evaluated the gauge coupling at the same momentum scale as the gaugino mass.

Although this is one-loop (and there is an additional 1/6 suppression), there are factors which can make it large. First of all, we see that there is a logarithm; if there is a large mass splitting, there will be a corresonding enhancement. Second, there is a multiplicity factor; "every" particle with gauge charge contributes. This last fact was also what contributed to the importance of standard electroweak parameters. However, the logarithm is different here because decoupling works differently than for standard electroweak corrections. Heavy scalar superpartners contribute more rather than less.

If we call the soft scalar mass $m_{0}$ and the coefficient of the trilinear scalar coupling $A$, for the standard oblique corrections (eg the $S$ parameter), it is easy to see that

$$
\Delta S \propto \frac{m_{L R}^{2}}{m^{2}} \sim \frac{\left(\lambda_{q} A m_{q}\right)^{2}}{m_{0}^{2}}
$$

which decouples for large $m_{0}$.

In the case of the SUSY-oblique corrections which we discuss, one finds

$$
\delta g(k) \propto \log \frac{m_{\tilde{q}}^{2}}{m_{q}^{2}}
$$

(where we now assume the quark mass is bigger than the gaugino's) which clearly does not decouple, and is in fact logarithmically enhanced. This logarithm can however be readily understood 6 . If we assume a chiral multiplet in which the scalar partner is heavy, there is an energy regime between the fermion (or gaugino) mass and the heavy scalar mass in which the gauge and 
gaugino coupling run differently. In fact one can also determine that when the scalar is the heavy partner the gauge coupling at low energy will be reduced relative to the gaugino couplings due to the sign of the fermion contribution to the beta-function for the gauge coupling.

Decoupling does occur however for SUSY-oblique corrections when $m_{q} \sim$ $m_{\tilde{q}}$

$$
\delta g \propto \frac{\left(m_{\tilde{q}}^{2}-m_{q}^{2}\right)}{m_{q}^{2}}
$$

Notice here that we have assumed the squark and quark to be nearly degenerate and heavier than the gaugino. Therefore what is relevant to SUSY-oblique corrections are standard model gauge charge multiplets with large supersymmetry breaking splitting in the masses. We conclude that heavy scalar partners can lead to interesting deviations from supersymmetric prediction $\delta g=0$.

\section{Large Effects and FCNC}

It is reasonable to ask whether there is any reason to believe that there might be large mass splittings, or nonstandard model gauged states. The answer might be yes, given the current ideas for resolving the flavor-changing-neutralcurrent (FCNC) problems in supersymmetric theories. The apparent degeneracy of squarks or alignment with quarks is still not understood. Potential explanations in the literature inglude gauge-mediated models and symmetries. It has also been pointed out 80 that the problem is ameliorated in models in which the first two generations of squarks are heavy. One example of a class of theories for which this is the case is the "More Minimal Supersymmetric Standard Model", in which one attempts to allow the maximal masses consistent with naturalness bounds. This permits the first two generations of squarks to be heavier which goes a considerable way towards solving flavor changing problems. One can then imagine $\tilde{m}_{1,2} \sim 20 \mathrm{TeV}, \tilde{m}_{3} \sim 100 \mathrm{GeV}-1 \mathrm{TeV}$.

A previous paper had also incorporated the idea that the first two generations are heavy. Specifically, they incorporated an anomalous U(1) symmetry as a mediator of supersymmetry breaking, and imagined that the first two generations were charged whereas the third generation was not. Squarks which are charged under $U(1)$ group can have mass of order $4 \pi$ times as big as usual supergravity induced mass because they get their mass from the larger $D$-term.

Another example in which there might be large effects are some models of gauge-mediation. Gauge mediation models can readily produce heavier squarks than gauginos since a gaugino mass requires $R$-symmetry breaking. An example of this was a class of models called "Mediator Models" 10 . The idea behind these models is that there is a weakly gauged global symmetry 
$G_{m}$ in the dynamical supersymmetry breaking sector and a SUSY breaking gaugino mass $M_{m}$. In addition, there are "mediator" fields which communicate with both sectors; specifically, one can have a vector-representation of both groups $T(m, 5), \bar{T}(\bar{m}, \overline{5})$ with a mass $M_{T} T \bar{T}$. These models have the advantage that there are no singlets, no complicated superpotential, and the correct vacuum is global minimum. However, gaugino masses arise at three loops whereas squark masses arise at two. The generic prediction then is that $m_{\tilde{q}} \sim 10 m_{1 / 2}$. This imposes naturalness problems but can yield interesting experimental signatures so it is testable.

A final example in which effects might be large is if there is a messenger sector without great mass splitting but with a large representation. That is, models in which there are many objects carrying the gauge charge can also yield big effects.

\section{Parameters and Numbers}

One can now ask for the kinds of models described above, what is the magnitude of the effects on gauge vs. gaugino coupling. There are three measureable quantities associated with $g_{3}, g_{2}, g_{1}$. These fit in well with a modified Peskin-Takeuchi naming convention $v=\left(g_{3}-\tilde{g_{3}}\right) / g_{3}, w=\left(g_{2}-\tilde{g_{2}}\right) / g_{2}$, $y=\left(g_{1}-\tilde{g_{1}}\right) / g_{1}$. Of course, one can use alternative names such as $\epsilon_{4}, \epsilon_{5}, \epsilon_{6}$.

We now calculate the numbers for each of these quantities for the models described above in which there is a large mass splitting. In reality, when the splittings are described as above in the gauge mediated case, one needs to be careful to run the parameters. We make the simple assumptions below to get an idea of the magnitude of the effects.

We define a mediator model spectrum by $m_{\tilde{l}}=10 m_{\tilde{w}}, m_{\tilde{q}}=20 m_{\tilde{w}}$. For this spectrum, we find $v=-10.5 \%, w=-3.4 \%, y=-1.7 \%$.

If we assume that sleptons are light and it is only the squarks which are heavy (probably necessary for these quantities to be measurable), one would calculate $w=-2.7 \%, y=-1.6 \%$.

We define a More Minimal Spectrum by $b_{R}, u, d, s, c, e, \mu, H$ having mass $20 \mathrm{TeV}$, whereas $b_{L}, t, \tau: 1 \mathrm{TeV}, m_{\tilde{w}}=100 \mathrm{GeV}, m_{\tilde{g}}=200 \mathrm{GeV}$. In this case, one obtains $v=-16 \%, w=-5 \%, y=2.6 \%$. For a modified more minimal model in which the sleptons have mass $1 \mathrm{TeV}$ instead, one obtains $w=-4.8 \%$, $y=-2.1 \%$.

\section{How to Measure?}

It is clear that there can be fairly sizable effects. It is therefore extremely interesting to ask the question whether these parameters are conceivably mea- 
surable. These numbers serve as benchmarks for interesting measurements, although a measurement at any level of accuracy will constrain our confidence in supersymmetry or equivalently constrain extensions of the minimal scenario.

Several groups have begun to investigate this question. Initially this was pursued as a test of supersymmetry at tree level11 but it was later pursued also in order to extract squark masses if they are heavy 6 .

The most precise measurement which has been suggested is of the $\mathrm{U}(1)$ coupling in a paper by Nojiri, Fujii, and Tsukamoto 6 where they studied $\sigma\left(e^{+} e^{-} \rightarrow \tilde{e}_{R}^{+} \tilde{e}_{R}^{-}\right)$. This process proceeds through $s$-channel gauge exchange and $t$-channel neutralino exchange. One can measure $\tilde{g}_{1}$ and $M_{1}$ by measuring the differential cross section $\frac{d \sigma\left(e^{+} e^{-} \rightarrow \tilde{e}_{R}^{+} \tilde{e}_{R}^{-}\right)}{d \cos \theta}$ with accuracy at $1 \%$ level. It has also been suggested by Cheng, Feng, and Polonsky to look at the $e^{-} e^{-}$ scattering cross section when running the collider in the appropriate mode, which can lead to even greater precision 112 . It should be borne in mind that although the $\mathrm{U}(1)$ coupling can be most accurately measured, the magnitude of $y$ is the smallest of the three precision measurements. It is therefore worthwhile to investigate the possibility of measuring $w$ and $v$ as well

Two possible ways to measure $w$ have been suggested 11 . If both charginos can be produced at the NLC, the gaugino coupling can be tested through the chargino mass dependence on the $H \tilde{H} \tilde{W}$ vertex. This only permitted about a 15-30\% measurement. In the "gaugino" region, where charginos are very nearly pure gauginos, one can test the $e \tilde{\nu} \tilde{W}$ vertex directly and $w$ can be measured to about $15 \%$. However, with independent measurement of $m_{\tilde{\nu}}, w$ can be measured to better than $5 \%$.

Finally we consider the measurement of $v$, which requires measuring the $\mathrm{SU}(3)$ gaugino Yukawa coupling. It is very likely that the best test will be at a hadron collider. At an $e^{+} e^{-}$collider, it might be possible to test the MMSSM if $\tilde{t}, \tilde{g}$ are sufficiently light ${ }^{g}$ or if $\tilde{b}, \tilde{g}$ are sufficiently light 1 One can then measure the cross section for $t \tilde{t} \tilde{g}$ production or $b \tilde{b} \tilde{g}$ production. For $b$ quark and squark production one predicts a few hundred events according to the gluino and squark mass (below a few hundred $\mathrm{GeV}$ ) and roughly half this number for tops. Details will be presented in a future paper1. Especially in the first case, but possibly in the second, it is also possible that the masses are such that the intermediate squark state is on shell, in which case one is in fact measuring the branching fraction into quark and gluino, which can also determine the gaugino coupling. The number of events we presented here is the production cross section but it is not corrected for cuts necessary to remove background events. Nonetheless for sufficiently light gaugino and third

${ }^{b}$ We thank Michael Peskin for suggesting this possibility

${ }^{c}$ We thank Jonathan Feng for pointing out this alternative possibility. 
generation squarks, it seems likely that a measurement of the gaugino coupling at at least the $15 \%$ level should be possible.

\section{More Non-Standard Physics}

Before concluding, we wish to stress the role of these measurements as not only a test of the parameters of the minimal extension of the standard model but also as an exciting probe of new physics. We have already stressed the fact that heavy squarks will lead to larger effects. This is especially important as it is precisely when the squarks become kinematically inaccessible that their virtual effects become largest. If one does not find the squarks, or if they are at the edge of the kinematically accessible regime, one would like to be able to confirm their existence and attempt some estimate of their mass through their virtual effects.

However, it might be that the squarks are relatively light, and one still measures large deviations from the supersymmetric relations. Provided they are not too large, one might ask how this could be consistent with supersymmetry. In fact, this is probably the most exciting possibility. It would indicate the existence of nonstandard fields which participate in supersymmetry breaking and therefore have a large mass splitting relative to the mass of the fermions or scalars and which carry standard model gauge charge. An obvious candidate in terms of existing models is a messenger sector, although possibilities extend beyond this. As an example, suppose the messenger sector consisted of a large vectorlike representatin under SU(5). One should note that for the "standard" messenger scenario, with a singlet coupled to messengers through a Yukawa term, the masses of the scalars are $\lambda^{2} S^{2} \pm \lambda F_{S}$ whereas the fermion mass is $\lambda S$. It is readily checked in this case that the logarithm takes a sign opposite to that before. We note that we need a nontrivial mass splitting in this nonstandard sector to get a sizable contribution. This would indicate a strong participation of this nonstandard model sector in supersymmetry breaking and would give great insight into the high-energy world.

\section{Conclusions}

To conclude, it is obvious that we would like to gain as many handles into the underlying supersymmetric theory as can be accessible. SUSY-Oblique corrections provide a different perspective into the physics of supersymmetry breaking and into the consistency of the standard supersymmetric sector. In nonstandard models, especially those addressing the supersymmetric flavor problem, it is likely that the parameters we have described can be large. Although it is almost certain that they will be a challenge to measure at a 
very interesting level. Clearly, the more precisely they are measured, the more constrained will be the underlying physics and our confidence in the supersymmetric model which is revealed through mass measurements. It is definitely worthy of further investigation.

\section{Acknowledgments}

We thank Jonathan Feng, Ian Hinchliffe, and Michael Peskin for conversations about measuring $v$. This research is supported in part by DOE under cooperative agreement \#DE-FC02-94ER40818, NSF Young Investigator Award, Alfred P. Sloan Foundation Fellowship, DOE Outstanding Junior Investigator Award.

While presenting this work, we learned of related research by Cheng, Feng, Polonsky, and Pierce, Nojiri, and Yamada 12.13.

1. E. Katz, L. Randall, and S. Su, MIT-CTP-2646

2. M. Golden and L. Randall, Nucl. Phys. B 361, 3 (1991).

3. M. Peskin and T. Takeuchi, Phys. Rev. Lett. 65, 964 (1990).

4. M. Peskin and T. Takeuchi, Phys. Rev. D 46, 381 (1992).

5. G. Altarelli and R. Barbieri, Phys. Lett. B 253, 161 (1991).

6. M. M. Nojiri, K. Fujii, and T. Tsukamoto, Phys. Rev. D 54, 6756 (1996).

7. A. Cohen, D. Kaplan, and A. Nelson Phys. Rev. Lett. 78, 2300 (1997).

8. G. Dvali and A. Pomarol, Phys. Rev. Lett. 77, 3728 (1996).

9. S. Dimopoulos and G. Giudice Phys. Lett. B 357, 573 (1995).

10. L. Randall, hep-ph/9612426.

11. J. Feng, H. Murayama, M. Peskin, and X. Tata Phys. Rev. D 52, 1418 (1995).

12. H.-C. Cheng, J. Feng, N. Polonsky, hep-ph/9706438

13. D. Pierce, M. Nojiri, and Y. Yamada, SLAC-PUB-7558, in preparation. 